sphenoid sinus. This therapy will help prevent complications in the future.

doi:10.1017/S002221511600640X

\section{ID: IP144}

\section{Management for temporal bone cancer: A} single institution experience

\section{Presenting Author: Shinya Morita}

Shinya Morita, Akihiro Homma, Yuji Nakamaru, Tomohiro Sakashita, Atsushi Fukuda, Takatsugu Mizumachi, Satoshi Fukuda

Hokkaido University Graduate School of Medicine

\section{Learning Objectives:}

Objective: We aimed to evaluate the prognostic factors and efficacy of treatment modalities for patients with temporal bone cancer, and to determine if definitive chemoradiotherapy (CRT) for advanced-stage disease can provide a substitute for highly invasive surgeries.

Methods: We performed a retrospective, single-institution review of 66 patients with previously-untreated squamous cell carcinoma of the temporal bone treated with curative intent between April 1997 and March 2015. Lateral temporal bone resection was selected as the initial choice of treatment for patients with T1-2 tumors. If histologic examination revealed positive surgical margins or more extensive involvement than preoperative imaging suggested, postoperative radiotherapy (RT) or CRT was performed. For patients with $\mathrm{T} 3-4$, lateral or subtotal temporal bone resection was performed. Adjuvant RT or CRT for T3-4 disease was systematically proposed after surgical resection. For locally advanced tumors with extension to the pyramidal apex, posterior cranial fossa, middle cranial fossa or internal carotid artery, definitive CRT or RT was also selected.

Results: The 5-year overall survival (OS) rate for each T classification was $100 \%$ for $\mathrm{T} 1,76.2 \%$ for $\mathrm{T} 2,55.6 \%$ for $\mathrm{T} 3$ and $36.7 \%$ for T4. Univariate and multivariate analysis showed that $\mathrm{T}$ classification was an independent predictor of the OS rate (hazard ratio 5.64; 95\% confidence interval 1.34-23.8; $p=0.0184$ ). Analysis by treatment modality revealed that the 5-year OS rate for patients with T1-2 was $100 \%$ for surgery and $81.3 \%$ for RT alone. The rate for patients with T3-4 was $52.1 \%$ for definitive CRT and $55.6 \%$ for surgery followed by RT with or without chemotherapy.

Conclusions: Patients with T1-2 benefited from surgical intervention without significant morbidity or mortality. Our findings also suggested that definitive CRT might be appropriate as the first-line treatment for T3-4, especially in cases with unresectable tumors.

doi:10.1017/S0022215116006411

\section{ID: IP145}

\section{Recurrent cases in pediatric congenital cholesteatoma}

\section{Presenting Author: Yuka Morita}

Yuka Morita $^{1}$, Kuniyuki Takahashi ${ }^{2}$, Shinsuke Oshima ${ }^{2}$, Shuji Izumi ${ }^{2}$, Yamato Kubota ${ }^{2}$, Yutaka Yamamoto ${ }^{3}$, Sugata Takahashi ${ }^{2}$, Arata Horii ${ }^{2}$

${ }^{1}$ Niigata university, ${ }^{2}$ Niigata University, ${ }^{3}$ Jikei

University

\section{Learning Objectives:}

Congenial cholesteatoma in children is a challenging condition because complete resections sometimes difficult and it often recurs. There are two types of recurrence: residual cholesteatoma and the retraction cholesteatoma. It is very important to know the risk factors of recurrence for the management of congenital cholesteatoma. For this purpose, we compared various factors between the recurrence $(+)$ group and (-) group.

Medical records of 67 children under 15 years old who underwent surgery for tympanic type of congenital cholesteatoma from 1999 to 2012 were retrospectively reviewed. Not only cholesteatoma found after onestage surgery but also found during or after second-look operation was defined as recurrence. Because pathophysiology of residual cholesteatoma and retraction cholesteatoma may be different, we investigated risk factors of each type of recurrence separately. Factors compared between the recurrence $(+)$ and $(-)$ groups are age, gender, and stage according to the Potosic classification.

Residual cholesteatoma and retraction cholesteatoma was seen in 14 ears $(20.8 \%)$ and $4(6 \%)$ ears out of 67 ears, respectively, and 49 patients had no recurrence. There was no significant difference in age and gender between the residual recurrence $(+)$ and recurrence $(-)$ group. However, patients with advanced stage of Potosic classification had more residual recurrence than no recurrence group $(\mathrm{p}=0.004)$. All cases of retraction cholesteatomas were Potosic stage IV, male and age at surgery was $4 \pm 0.8$, which was significantly younger than recurrence $(-)$ patients $(6.4 \pm 3.6)$.

Advanced stage was the significant risk factor for residual cholesteatoma. In contrast, younger patients with advanced stage were the risk factors of retraction recurrence. In surgeries for younger patients with advanced stage, extensive removal of mucosa as well as epithelium have to be performed in young patients with developing tubal function, which might result in retraction recurrence. 